\title{
First patellar dislocation: from conservative treatment to return to sport
}

\author{
STEFANO RESPIZZI, RAMON CAVALLIN
}

Department of Rehabilitation and Functional Recovery, IRCCS Istituto Clinico Humanitas, Rozzano (MI), Italy

\begin{abstract}
Treatment of first patellar dislocation is usually conservative and the subsequent rehabilitation program is based on specifically formulated objectives, which can be divided into different stages: stage 1: resolution of pain, swelling and inflammation; stage 2: recovery of joint motion and flexibility; stage 3: recovery of muscle strength; stage 4: recovery of motor patterns and coordination; and stage 5: recovery of the sportspecific athletic action and return to sporting activity. The aim, in the management of a patient affected by first patellar dislocation, is to achieve the best possible functional recovery: since this is a condition often affecting young athletes, this means returning them to their pre-injury conditions, both in terms of fitness and the level of sporting activity practiced. By proceeding through functional stages, the risk of recurrence of the dislocation can be reduced. The "go-ahead" to resume sporting activity can only be given in the presence of normal results on sport-specific functional tests.
\end{abstract}

Key words: athlete, dislocation, patella, rehabilitation, sport.

\section{Introduction}

The management of patella femoral instability is particularly complex (1-2). Despite the plentiful literature on this topic, robust scientific data are often lacking.

\footnotetext{
Corresponding Author:

Stefano Respizzi, MD

Department of Rehabilitation and Functional Recovery,

IRCCS Istituto Clinico Humanitas

Via Manzoni 56, 20089 Rozzano (MI), Italy

E-mail: stefano.respizzi@humanitas.it
}

In this setting, evidence-based medicine, failing to provide confirmation of the therapeutic approaches that are suggested by experience and common sense, offers little help in making the best choices.

The estimated incidence of first dislocation of the patella is 5.8 new cases per 100,000 people, rising to 29 per 100,000 in the population aged between 10 and 17 years (3). The recurrence rate in patients treated conservatively ranges from 15 to $44 \%$. In addition to these patients, it is necessary to consider those subjects who, while not displaying frank dislocations, never the less continue to experience pain and mechanical dysfunction following a first dislocation (4).

The mechanisms leading to patellar dislocation can be direct or indirect. Direct traumas arise from a sudden mechanical force exerted on the patella that causes it, in most cases, to shift laterally. The indirect mechanism (more common) mainly occurs when the foot is fixed on the ground and the knee is subjected to valgus stress and internal rotation of the femur or external rotation of the tibia. The direction of dislocation is usually medial-to-lateral and simple extension of the knee is often enough to reduce the dislocated patella.

Treatment is usually conservative and the subsequent rehabilitation program is based on specifically formulated objectives, which can be divided into different stages (5):

- Stage 1: resolution of pain, swelling and inflammation

- Stage 2: recovery of joint motion and flexibility

- Stage 3: recovery of muscle strength

- Stage 4: recovery of motor patterns and coordination

- Stage 5: recovery of the sport-specific athletic action and return to sporting activity.

These stages should not be considered a rigid scheme 
but rather a general direction to follow, bearing in mind that the different stages may overlap and that a patient's progress, depending on his/her healing capacity, will be characterized by the achievement of increasingly complex functional objectives.

\section{Resolution of the immediate effects of the injury}

It is advisable to apply, as early as possible, the RICE rule (i.e. Rest, Ice, Compression, Elevation), which can be associated with the administration of non-steroidal anti-inflammatory drugs (NSAIDs).

To reduce post-traumatic swelling it is certainly useful to perform lymphatic drainage massages of knee. It is common in this stage to have recourse to physical therapies in order to reduce swelling and pain, both of which are factors that negatively affect muscle activation.

First of all, the medial patella femoral structures need to be kept in a shortened condition to encourage their healing process. This requires a certain degree of immobilization, which can be achieved using specific braces or functional bandaging for a period of 2-3 weeks. In this way, the recovery is speeded up and the risk of stiffness is reduced. In our view, the taping method proposed by McConnell also seems valid (6). Offering the same advantages as or theses and early mobilization, it seems to guarantee more stable shortening of the damaged structures, thanks to the fact that it is tailored to the single patient. Walking is allowed with two crutches and with as much loading as is tolerated.

\section{Recovery of joint motion and strength}

This stage has several aims: to recover knee joint motion, to promote activation and strengthening of the vastus medialis obliquus (VMO), to re-establish a correct gait pattern, and to introduce proprioceptive exercises (7). In this stage it is a good idea to continue supporting the stabilization of the patella through the use of braces or taping. It is advisable to begin mobilization cautiously, also making use of neuromuscular facilitation techniques such as muscle contraction and release below the pain threshold, initially with the patient seated on a high couch; once a moderate recovery of joint motion has been achieved and the swelling has reduced appreciably, the same technique can be applied with the patient prone. In this stage it is useful to use a cycle ergometer, getting the patient to forward pedal and back pedal through a pain-free range of motion.

Efforts to achieve muscle strengthening and recovery of correct patterns of neuromuscular activation are, as already indicated, fundamentally important; accordingly, our work focuses mainly on the VMO and the glutei. Individuals with patellar instability frequently show generalized ligament laxity and therefore need particularly effective control in order to prevent new dislocations. Electrical stimulation or biofeedback training of the VMO are useful for recovering, as early as possible, control of the patella (Fig. 1).

Closed chain knee extension exercises deserve particular attention. Moreover, closed kinetic chain exercises also allow training, at the same time, of the gluteal muscles and the trunk stabilizers, thereby encouraging postural synergies.

When choosing the type of exercise to use, especially in the immediate post-acute phase, it is important to bear in mind a few concepts relating to patella femoral stress, which shows different trends during knee movement performed in closed kinetic chain versus open kinetic chain exercises. In open kinetic chain

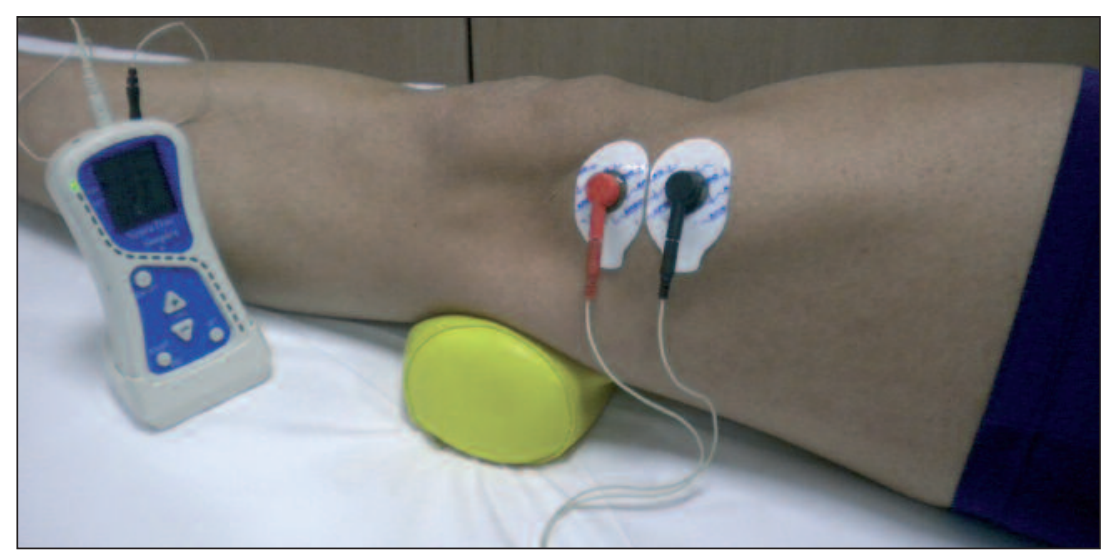

Fig. 1. Strengthening the vastus medialis obliquus using biofeedback training. 
activities, $10^{\circ}-0^{\circ}$ and $90^{\circ}-50^{\circ}$ ranges of motion can be considered safe, while $50^{\circ}-0^{\circ}$ can be considered a safe range in closed chain activities.

In addition, these subjects also seem to show impaired femoral control, resulting in adduction and internal rotation. In particular, reduced generation of force by the glutei, abductors and external rotators of the hip and delayed activation of the gluteus medius have been detected (Fig. 2). Strengthening the gluteal muscles and trunk stabilizers is necessary in order to increase the stability of the lower limb during the stance phase of walking and the heel strike phase of running, therefore they must also be trained in loaded conditions (Fig. 3).

It is important to begin specific gait training early, since these subjects often develop gait abnormalities as a defense mechanism against patellar instability. Indeed, they may adopt two strategies: keeping the knee extended for as long as possible during the stance phase, or avoiding complete extension of the knee, so that the patella does not go through the range of motion in which it is particularly vulnerable to dislocation, i.e. the first $20^{\circ}-30^{\circ}$ of knee flexion.

It is also essential to re-educate the patient to maintain correct alignment of the lower limb when climbing or going down stairs. This is done with the help of a mir- ror, beginning with a small number of slow, correct executions and then gradually increasing the speed (Fig. 4). As the training progresses, the rehabilitation program can be extended, adding forward and backward walking on a treadmill and water running, and finally slow running on the treadmill to begin aerobic reconditioning.

\section{Functional recovery}

This is the stage in which all that has been achieved in terms of joint motion, muscle strength and endurance and proprioception should be translated into sportrelated actions (5). Running activities are increased, and changes of direction, different speeds and crosssteps are introduced. At the same time plyometric exercises are added and isotonic muscle work and aerobic conditioning are continued. Isokinetic training at low and high angular velocities can also be introduced. Knee function can be evaluated using the figure-ofeight test and the stair-running test.

In the figure-of-eight test, the subject is timed as he/she runs three times around two circles, each with a diameter of 4 meters, each time tracing the shape of a figure 8. In the stair-running test, the subject is

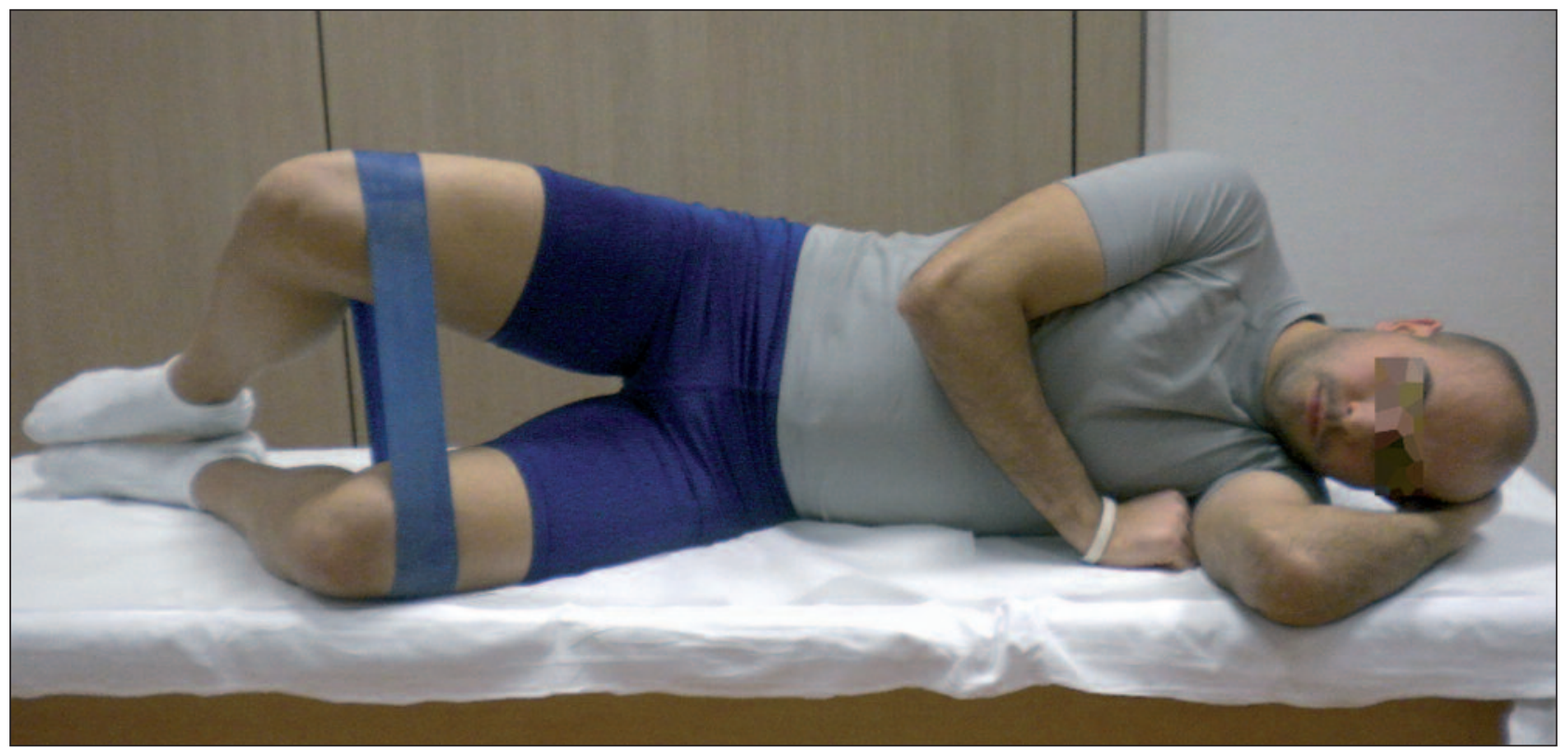

Fig. 2. Strengthening of the abductors and external rotators of the hip in unloaded condition. 

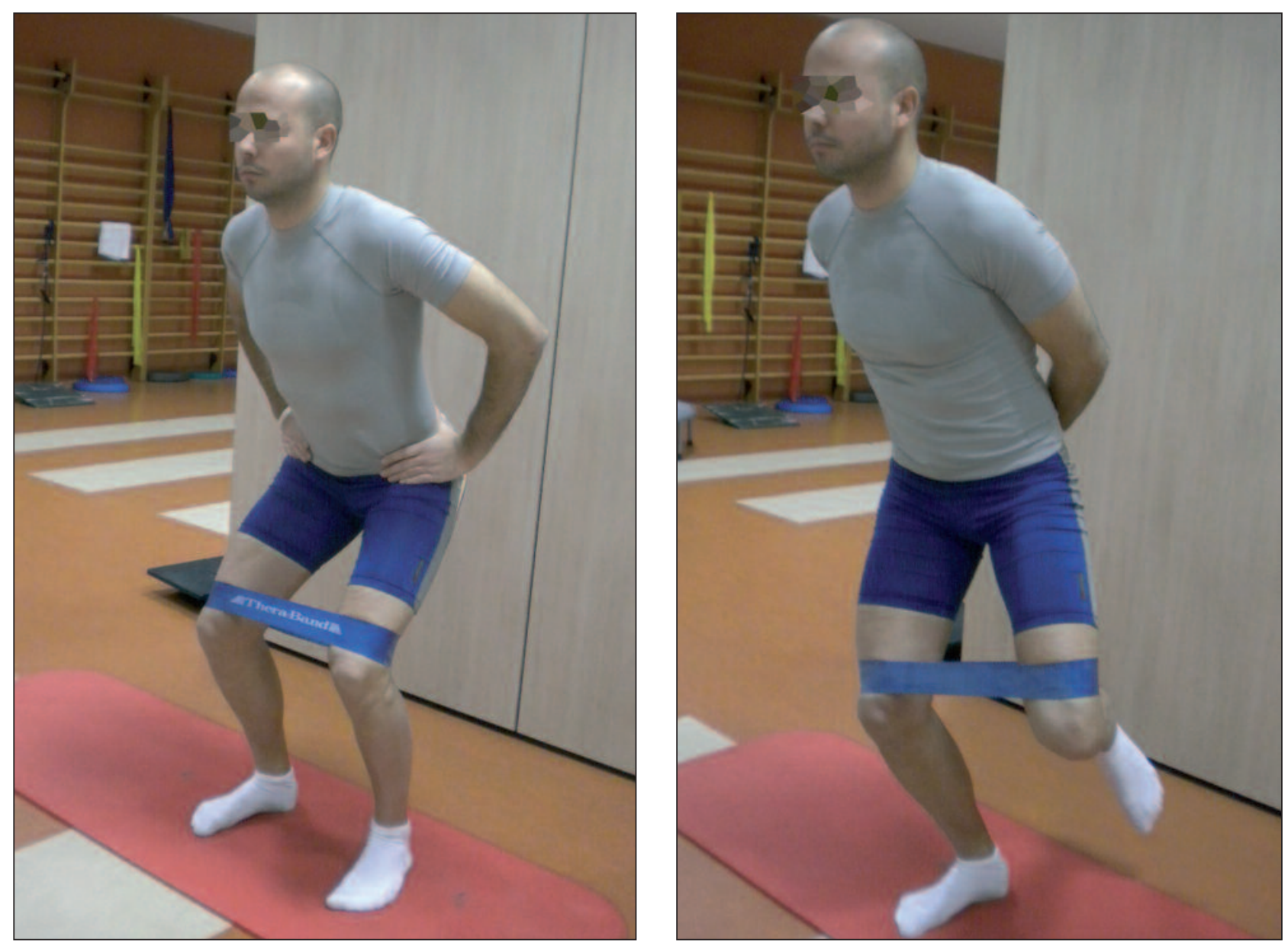

Fig. 3. A: Strengthening of the abductors and external rotators of the hip with loading of both legs. B: Strengthening of the abductors and external rotators of the hip with loading of one leg.

required to run up and down a 55-step staircase, again being timed as he/she does so.

A series of three functional performance tests (FPTs) can be used to establish a subject's fitness to perform an athletic or sporting action: co-contraction test, carioca test and shuttle run test.

In the first test, the subject is required to do a series of jumps along a semi-circle drawn on the floor while attached to the wall by an elastic cord. According to the test protocol, he/she must cover five semi-circles in the shortest possible time. In the carioca test, the subject moves sideways, taking cross-steps. Finally, in the shuttle run test, the subject runs a 6.1-metre distance four times, stopping and changing direction at the end of each sprint.
For each test, three trials are performed and timed; the best times are summed to obtain the subject's FPT score. In anticipation of the subject's return to competitive sport, it is a good idea to conduct a series of monopodalic tests, such as: single leg hop test, triple leg hop test and side jump test. These tests, which have been defined valid indicators of strength and functional stability, demand explosive power, coordination and balance (in a nutshell, "neuromuscular control"). The subject also needs to be confident of being able to accomplish them without particular risks.

In the single leg hop test the subject hops once on one leg, whereas in the triple leg hop test he/she is required perform a series of three consecutive hops on one leg; both legs are tested and the distance covered is 


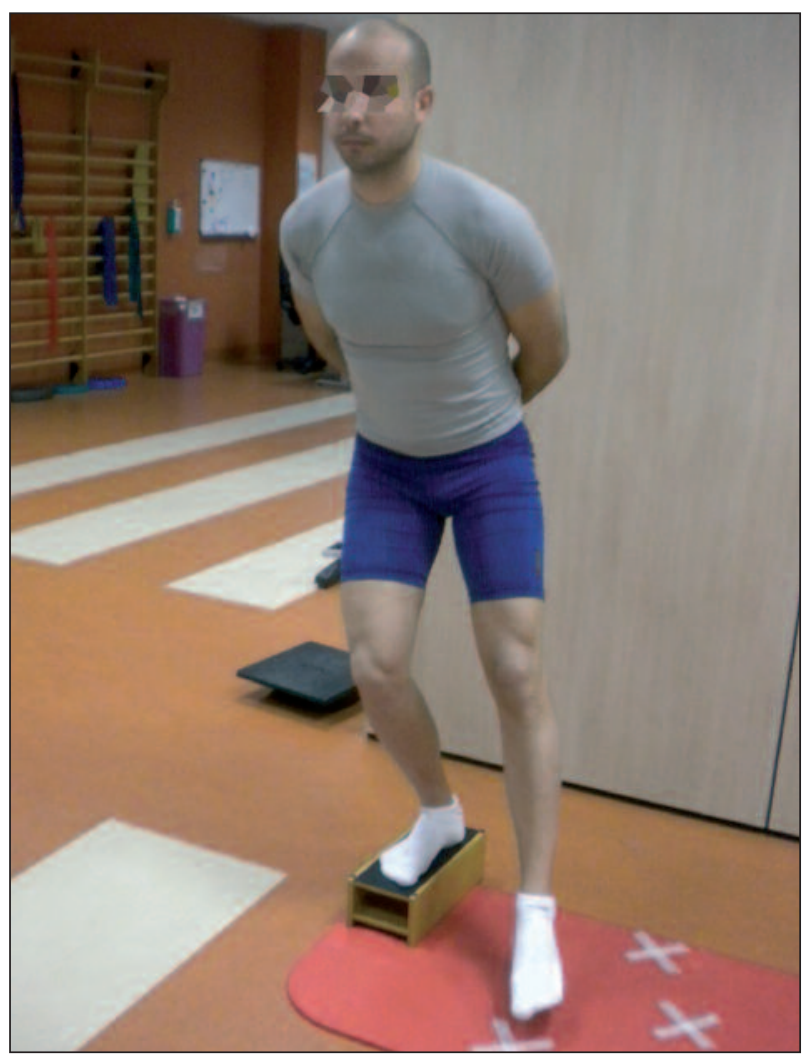

Fig. 4. Maintenance of correct lower limb alignment during proprioceptive exercises. measured. A maximum 10\% difference between the two legs is considered compatible with resumption of competitive sport. In the side jump test, the knee is subjected to stress not only in the sagittal plane, like the previous two tests, but also in the transverse plane. This test thus constitutes a more reliable assessment of functional recovery.

\section{References}

1. Fredericson M, Yoon K. Physical examination and patella femoral pain syndrome. Am J Phys Med Rehabil. 2006; 85:234-243

2. Fithian DC, Paxton EW, Stone ML, et al. Epidemiology and natural history of acute patellar dislocation. Am J Sports Med. 2004;32:1114-1121.

3. Colvin AC, West RV. Patellar instability. J Bone Joint Surg Am. 2008; 90: 2751-2762.

4. Hawkins RJ, Bell RH, Anisette G. Acute patellar dislocations. The natural history. Am J Sports Med. 1986;14:117-20.

5. Ricci G, Respizzi S. Ritorno allo sport dopo trattamento conservativo. Journal of Sports Traumatology. 2011;28:66-72.

6. McConnell J. Rehabilitation and nonoperative treatment of patellar instability. Sports Med Arthrosc. 2007; 15: 95-104.

7. Handfield T, Kramer J. Effect of Mcconnell taping on perceived pain and knee extensor torques during isokinetic exercise performed by patients with patella femoral pain syndrome. Physiother Can. 2000; Winter:39-44. 